\title{
Noise and Resolution of Bayesian Reconstruction for Multiple Image Configurations
}

\author{
Garry Chinn and Sung-Cheng Huang
}

\begin{abstract}
Images reconstructed by Bayesian and maximumlikelihood (ML) using a Gibbs prior with prior weight $\beta$ were compared with images produced by filtered backprojection (FBP) from sinogram data simulated with different counts and image configurations. Bayesian images were generated by the OSL algorithm accelerated by an overrelaxation parameter. For relatively low $\beta$, Bayesian images can yield an overall improvement to the images compared to ML reconstruction. However, for larger $\beta$, Bayesian images degrade from the standpoint of noise and quantitation. Compared to FBP, the ML images were superior in a mean-square error sense in regions of low activity level and for small structures. At a comparable noise level to FBP, Bayesian reconstruction can be used to effectively recover higher resolution images. The overall performance is dependent on the image structure and the weight of the Bayesian prior.
\end{abstract}

\section{INTRODUCTION}

$S^{T}$ TATISTICAL methods for image reconstruction have the potential of improving the quality of images compared to the filtered backprojection reconstruction. The reconstruction problem for emission tomography can effectively be described by a linear-Poisson (linear map, multi-variate Poisson) model from image space to projection space. This model provides a framework for incorporating the physical and non-stationary statistical properties of tomographic image reconstruction. For example, the intrinsic detector response and attenuation can be directly accounted for without employing correction schemes. The introduction of expectation-maximization algorithms by Shepp and Vardi [9] and Lange and Carson [4], have led to the development of feasible maximumlikelihood (ML) reconstruction algorithms. Since image reconstruction is ill-conditioned, regularization can improve the quality of the reconstructed images. Many different regularization methods have been proposed for $\mathrm{ML}$ reconstruction.

Manuscript received November 25, 1992; reviewed August 4, 1993. This work was supported by US DOE grant no. DE-FC0387ER60615 and NIH grants CA 56655, NS 15654, and MH 37916.

The authors are with the Division of Nuclear Medicine and Biophysics and the Crump Institute for Biological Imaging UCLA School of Medicine, 405 Hilgard Avenue, Los Angeles, CA 90024.

G. Chinn is also with the Department of Electrical Engineering UCLA School of Engineering and Applied Sciences, Los Angeles CA 90024.

This paper was originally presented at the 1992 Nuclear Science Symposium, held in Orlando, FL, October 25-31, 1992.

IEEE Log Number 9212530.
A feasibility stopping criterion was introduced by Veklerov and Llacer [11]. Snyder and Miller proposed suppressing the edge and noise artifacts of ML-based reconstruction through the use of Gaussian sieve regularization [10]. Alternatively, Bayesian methods using a "smoothness" prior have the potential advantage of preserving edges.

Because these techniques are non-linear, the performance of each is dependent on several factors including the size, shape and activity of structures in the phantom. Liow and Strothers [7], [8] have studied ML reconstruction with Gaussian sieves. Lalush and Tsui [3] evaluated Bayesian reconstruction with Gibbs priors and the effects of the prior strength and iterations. However, no attempt was made at studying the noise and resolution systematically. Characterizing the performance of different reconstruction algorithms will be important for evaluating current and future research. In this paper, we examine the noise properties, resolution and quantitation of Bayesian ML reconstruction with Gibbs priors for multiple image configurations.

\section{BAYESIAN RECONSTRUCTION}

Bayesian methods use an image before reweight the probability of certain image configurations. Geman and Geman [1] suggested using Gibbs priors for Bayesian image estimation. An energy function for an image sequence $\lambda_{j}, j=1 \cdots n$ where $n$ denotes the number of pixels, can be defined as

$$
U(\lambda)=\sum_{i, j} \phi\left(\lambda_{i}-\lambda_{j}\right)+\epsilon \sum_{k} \phi(\lambda),
$$

where $\phi$ is some potential function defined on pixel pairs or individual pixels. Increasing "roughness," results in increasing energy. It can be shown that the probability density function for a Markov random field can be expressed by the Gibbs distribution,

$$
P(\lambda)=\frac{\exp (-\beta U(\lambda))}{Z},
$$

where $\beta$ is the prior weight and $Z$ is the normalizing partition function,

$$
Z=\int_{\lambda} \exp (-\beta U(\lambda))
$$


The Bayesian estimate can now be found by maximizing the following $\log$ posterior function

$$
S(\lambda)=L(\lambda)-\beta U(\lambda)-\log Z .
$$

Geman and McClure [1] proposed using maximum-likelihood to estimate $\beta$ through an iterative technique. The drawback of their suggested approach is the very high computational cost. Furthermore, the iterative technique does not yield a closed-form solution.

A direct analytical solution to the ML $\beta$ estimation problem exists for the class of generalized Gaussian potential functions as suggested by $K$. Lange ([5] and personal communication). Let

$$
\phi(r)=|r|^{\alpha}, \quad \alpha>1 .
$$

In this case, we find that

$$
\beta U(\lambda)=U\left(\beta^{\alpha} \lambda\right) .
$$

Applying a change of variable and substituting (6) into (3) we find that

$$
Z=\beta^{-n / \alpha} \int_{\theta} \exp [-U(\theta)] d \theta
$$

where integration is over the $n$ dimensional (number of) pixel space. Substituting (7) into (4) results in a log posterior function

$$
L(\lambda)-\beta U(\lambda)+\frac{n}{\alpha} \log \beta .
$$

Therefore, the maximum likelihood estimate of $\beta$ given $\lambda$ can be solved for directly which yields

$$
\hat{\beta}_{M L}=\frac{n}{\alpha U(\lambda)} \text {. }
$$

Following the analysis of Lalush and Tsui [3], the derivative of the generalized Gaussian is given by

$$
\frac{\partial \phi(r)}{\partial r}=\alpha r^{\alpha-1}, \quad \alpha>1, \quad r \geq 0 .
$$

Thus for $1<\alpha \leq 2$, the behavior is similar to the $\log$-cosh potential of Green [2], smoothing out all differences above a certain $r$. The parameter $\alpha$ determines the cutoff level.

\section{Simulation Method}

Simulations were performed on a Sparcstation IPC with 80 projections to produce 64 by 64 images with $0.314 \mathrm{~cm}$ pixels. Each projection was sampled at $0.314 \mathrm{~cm}$ intervals with a detector resolution of $0.75 \mathrm{~cm}$ full width at half maximum (FWHM) and Poisson noise. The simulation set consisted of the Hoffman brain phantom and 3 cylinder phantom. The Hoffman brain phantom had a 4:1 activity ratio between gray and white matter. The background for each cylinder phantom was a low activity cylinder of radius $7 \mathrm{~cm}$. Three high-activity cylinders $(r=2 \mathrm{~cm})$ were symmetrically placed in phantom 1 and 2 . The high-activity cylinders in phantom 3 had radii of $0.5,1.0$ and $2.0 \mathrm{~cm}$.
The high-low activity ratio was $4: 1$ for phantoms 1 and 3 , and was $2: 1,3: 1$ and $4: 1$ in phantom 2 . For each phantom, $L=40$ independent trials were simulated for both high $(200 \mathrm{~K})$ and low $(10 \mathrm{~K})$ count conditions.

The bias can be estimated for each pixel from

$$
b(j)=\operatorname{mean}\left[\hat{\lambda}_{j}\right]-\lambda_{j},
$$

where,

$$
\operatorname{mean}\left[\hat{\lambda}_{j}\right]=\frac{1}{L} \sum_{l=1}^{L} \hat{\lambda}_{j}(l) .
$$

The estimated variance is given by

$$
\sigma^{2}(j)=\frac{L}{L-1}\left[\operatorname{mean}\left[\hat{\lambda}_{j}^{2}\right]-\operatorname{mean}\left[\hat{\lambda}_{j}\right]^{2}\right] .
$$

The bias and variance for each pixel reported in this paper are normalized against the true (known) pixel value and are referred to in the figures as the normalized bias and normalized variance. The effective global Gaussian resolution (EGGR) [7] can be defined as follows. Let $G(\mathrm{FWHM})=$ true image $*$ Gaussian (FWHM) where ' $*$ ' denotes the convolution operator. Then the EGGR is the FWHM that maximizes the spatial correlation function of the reconstructed image and $G$ (FWHM).

The OSL algorithm [2] with an acceleration factor $\gamma$ was used to reconstruct the Bayesian images. To stabilize acceleration, the OSL iteration sequence was dampened by a simple averaging technique. The OSL iterations were executed in multiples of three with the last two iterations averaged. Damping reduced the acceleration noise artifacts for ML reconstruction ( $\beta=0)$ as shown in Fig. 1.

For each phantom data set, the reference reconstruction method was filtered backprojection (FBP) using the following filters with cutoff $\left(F_{c}\right)$ expressed in $\pi$ radians: a) ramp 1.0 , b) Shepp-Logan 1.0, c) Shepp-Logan 0.8 , d) Shepp-Logan 0.6, e) Hanning 1.0, and f) Hanning 0.8. The Bayesian images were reconstructed using the generalized Gaussian potential function described earlier with $\alpha=1.01$ and $\epsilon=1.0$. This particular choice of $\alpha$ leads to a relatively flat $\partial \phi(r) / \partial r$ function and will tend not to preserve edge information in the reconstructed image. The OSL reconstruction kernel was chosen to be $0.75 \mathrm{~cm}$ FWHM to maximize resolution recovery. For the cylinder phantoms, 12 accelerated OSL iterations were used. For the Hoffman brain phantom, images were reconstructed using 12 accelerated EM iterations and 60 accelerated OSL iterations with various $\beta$.

\section{Results AND Discussion}

\section{A. Noise Structure}

Fig. 2 shows the mean, variance and bias images of the brain phantom at $200 \mathrm{~K}$ total counts reconstructed by ML and FBP using a Shepp-Logan filter with cutoff frequency $F_{c}=0.8$ of the Nyquist frequency $F_{N}$. In the case of ML-based methods, the variance is proportionally related to the underlying image structure. Fig. 3 better illustrates 


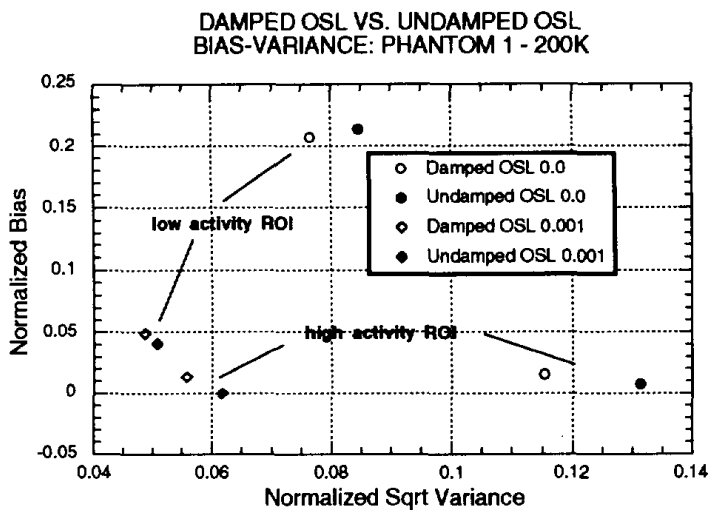

Fig. 1. Bias versus variance of images reconstructed from accelerated OSL algorithm with and without damping. Shown are the results for ML and Bayesian reconstruction for different high and low activity ROI in phantom 1 at $200 \mathrm{~K}$.
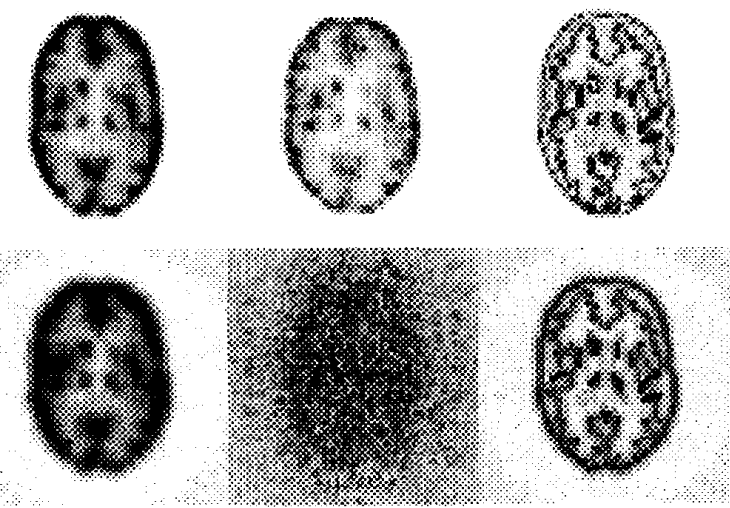

Fig. 2. Top Row (left to right): The mean, variance and bias images produced from $\mathrm{ML}$ reconstruction for the Hoffman brain phantom. Bottom Row (left to right): The mean, variance and bias images produced from FBP using a Shepp-Logan filter with cutoff 0.8 of the Nyquist frequency. Note that for ML images, the noise is dependent on the image structure.

this relationship for Bayesian-ML versus FBP. Shown in this figure is a pixel by pixel scatter plot of the mean versus variance for ML, Bayesian with $\beta=0.0002$ and FBP Shepp-Logan $F_{c}=0.8$. The image variance reflects the same properties of the Poisson noise. In contrast, FBP distributes the noise energy uniformly over the image support. This result is consistent with the observation that the ML approach has superior mean-square error performance compared to FBP in low activity regions.

\section{B. Noise versus Quantitation}

The normalized bias and normalized variance were computed from the average pixel values of different region of interests (ROI). The results are shown in Fig. 4. High activity ROI's consisted of 2 by 2 pixel blocks positioned near the center of high activity structures - high activity cylinders in the cylinder phantom and gray matter for the brain phantom. The low activity ROI's consisted of
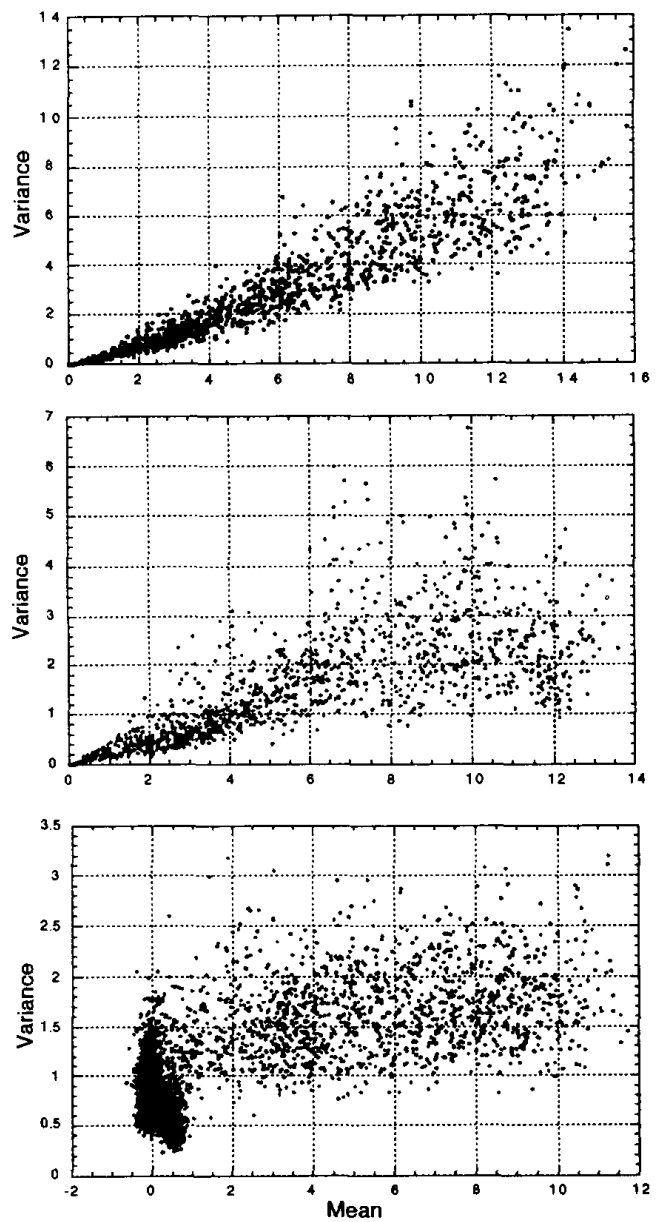

Fig. 3. The mean versus variance on a pixel by pixel basis of (top) ML reconstruction, (middle) Bayesian reconstruction $\beta=0.0001$, and (bottom) FBP Shepp-Logan $F_{c}=0.8$ of the Hoffman brain phantom.

the average of ten 2 by 2 pixel blocks randomly scattered within the large, low-activity cylinder for the cylinder phantom and white matter for the brain phantom. This provided a representative sample of the low activity regions for each phantom.

In the low activity region, the $\mathrm{ML}-$ Bayesian images have lower noise variance compared to FBP images for all image configurations. Within high-activity regions of the cylinder phantoms, the ML images have variance comparable to FBP Shepp-Logan with $F_{c}=0.6$. As a result, the absolute bias of ML images is better than the FBP ramp $F_{c}=1.0$ in the high-activity regions of the brain phantom. Also unlike the cylinder phantoms, the variance of $\mathrm{ML}$ images is higher than the FBP ramp $F_{c}=1.0$. Applying Bayesian smoothing does not yield improvements in the bias of high activity structures. Since the high activity ROI's are chosen within the center of structures (cylinders in the cylinder phantom and 'rims' in the brain phantom), the effect of the Bayesian prior on the overshoot at the rims is not shown directly in the bias-variance 

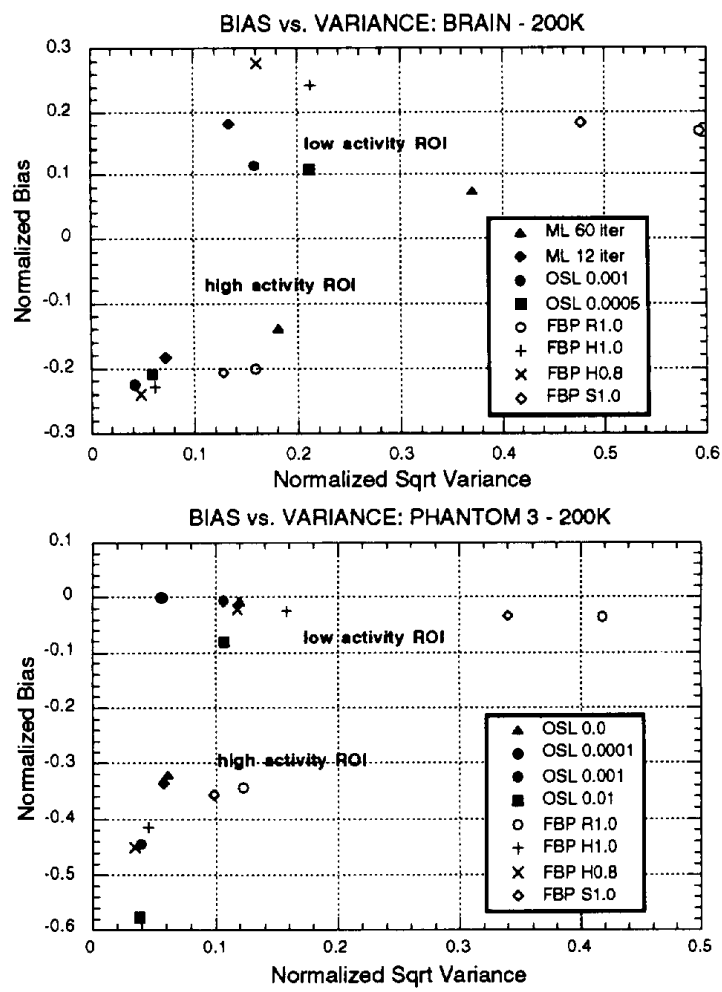

Fig. 4. Bias versus variance of images produced from the OSL algorithm. Shown are the results for the (top) Hoffman brain phantom and (bottom) cylinder phantom 3 each at $200 \mathrm{~K}$ total counts. The images were reconstructed with varying prior strength $\beta$ where 0.0 corresponds to the ML case. Also shown are the results of images produced by FBP with a ramp (R1.0), Hanning (H1.0, H0.8) and Shepp-Logan filters (S1.0, etc. ...) with various cutoff frequencies expressed as a fraction of the Nyquist frequency.

graph of Fig. 4. In the center of the high activity structures, we find that the smoothing effect of the Gibbs priors tends to increase the negative bias. However, smoothing reduces the noise variance to a level comparable in range to that of FBP Shepp-Logan $F_{c}=1.0$ at 200 $\mathrm{K}$ total counts.

The effect of varying the prior weight $\beta$ on the biasvariance of the images is illustrated in Fig. 5. In general, a small $\beta$ improves the quantitation of ML reconstruction. For increasing $\beta$, the image variance is also reduced, until $\beta$ passes some critical value, at which point the reconstruction begins to deteriorate. The critical $\beta$ is shown in Fig. 5. Table I lists the MLE $\beta$ values for each configuration. These values were calculated using (9) based on the true image configuration. In each case, we see that the MLE $\beta$ exceeds the critical $\beta$ where Bayesian images breakdown and the image bias-variance is no longer optimal.

\section{Noise vs. Resolution}

The EGGR results are shown in Fig. 6 for the brain phantom and cylinder phantom 3 at $200 \mathrm{~K}$ counts. Below the critical $\beta$, the MI-Bayesian images have better (lower

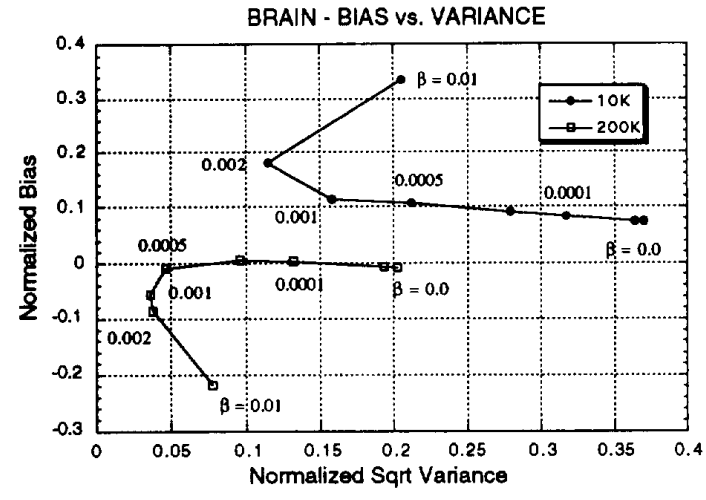

PHANTOM 1 - BIAS vs. VARIANCE

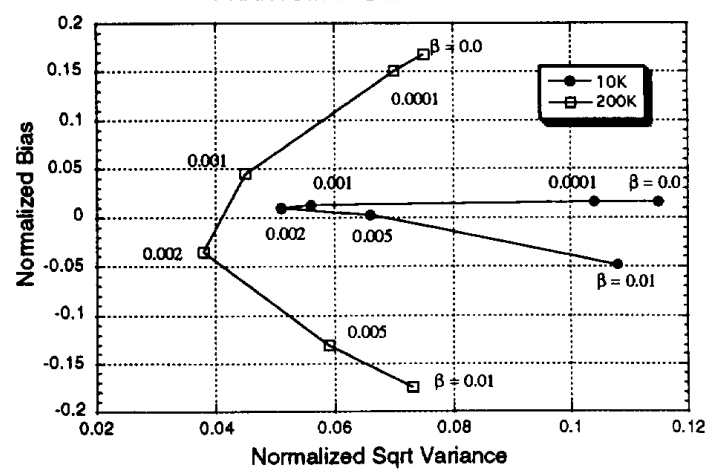

Fig. 5. The effects of increasing $\beta$ in Bayesian reconstruction. Shown is the bias versus variance of the (top) brain phantom and (bottom) cylinder phantom 1 for increasing $\beta$ in a low and high activity ROI.

TABLE I

MLE PRIOR WEIGHT

\begin{tabular}{lcc}
\hline & Low 10 K & High 200 K \\
\hline Phantom 1 & 0.084 & 0.0041 \\
Phantom 2 & 0.089 & 0.0043 \\
Phantom 3 & 0.087 & 0.0042 \\
Brain & 0.046 & 0.0022 \\
\hline
\end{tabular}

MLE $\beta$ for each image configuration used in this study.

EGGR) resolution compared with the FBP ramp $F_{c}=1.0$. For the brain phantom, the critical $\beta$ produces images with FWHM less than the value of the intrinsic resolution. However, stopping the accelerated OSL at 12 iterations produced images near the intrinsic resolution, suggesting that for low resolution recovery, fewer iterations of an ML algorithm may be preferred over Bayesian reconstruction.

\section{CONCLUSIONS}

Bayesian reconstruction using a Gibbs prior can improve the bias and variance of ML images at the sacrifice of resolution. For simple configurations it can produce images with superior quantitation, resolution and noise compared to conventional filtered backprojection. For more complex configurations, Bayesian methods can produce higher resolution images at a given noise level com- 

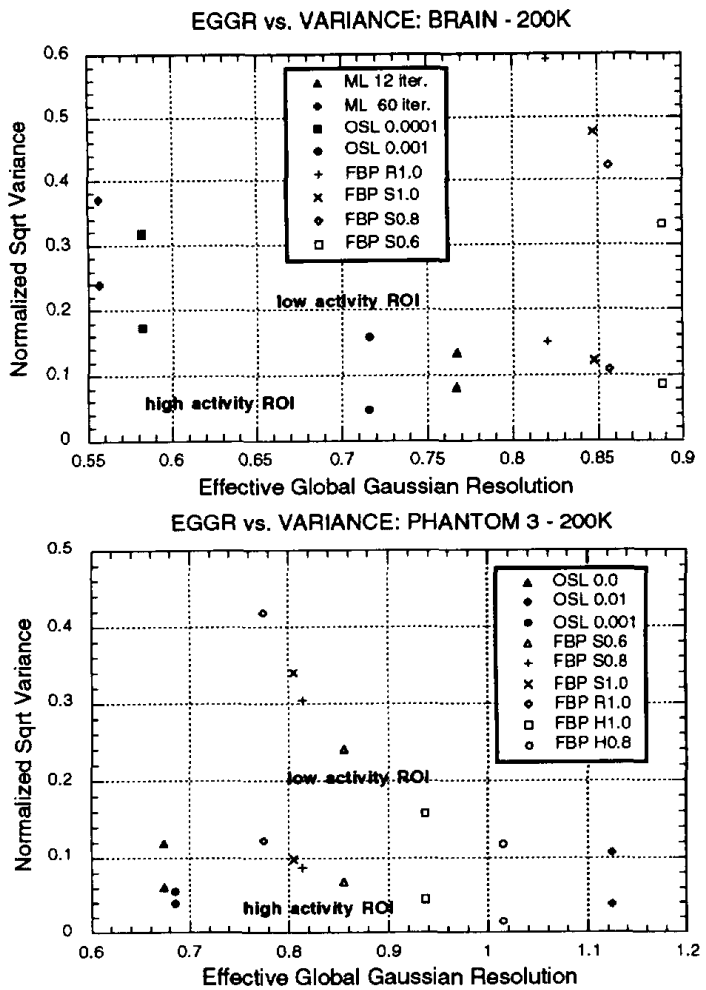

Fig. 6. Variance versus effective global Gaussian resolution (EGGR) from images produced by Bayesian reconstruction. The top graph shows the results for the Hoffman brain phantom at $200 \mathrm{~K}$. The bottom graph shows the results for cylinder phantom 3 at $200 \mathrm{~K}$. Images were produced with $\beta$ set from 0.0 to 0.01 . For phantom 3, 12, iterations were used while 60 iterations were used for the brain phantom. Images reconstructed by filtered backprojection using a ramp (R) and Shepp-Logan filters with $F_{c}$ at $1.0,0.8$, and 0.6 are also shown.

pared to filtered backprojection. Also the image noise variance of Bayesian ML images is proportional to the mean value, implying that the noise energy in high activity regions does not spread into adjacent low activity regions as it occurs with images reconstructed by filtered backpro- jection. We also demonstrated that the ML prior weight $\beta$ for the generalized Gaussian potential function, does not necessarily yield the optimal image in terms of bias and variance. Alternative techniques for selecting the weight $\beta$ and the number of iterations are needed.

\section{ACKNOWLEDGMENTS}

The authors would like to thank Dr. Magnus Dahlbom and Ms. Dan-Chu Yu for further software support, and also Dr. Kenneth Lange for many useful discussions on this topic.

\section{REFERENCES}

[1] S. Geman and D. E. McClure, "Statistical methods for tomographic image reconstruction," Bulletin de L'Institut Int'l de Statistique, vol. 52, pp. 5-21, 1987.

[2] P. J. Green, "Bayesian reconstruction from emission tomography data using a modified EM algorithm," IEEE Trans. Med. Imaging, pp. 84-93, Mar. 1990.

[3] D. Lalush and B. Tsui, "Simulation evaluation of Gibbs prior distributions for use in maximum a posteriori SPECT reconstructions," IEEE Trans. Med. Imaging, pp. 267-275, June 1992.

[4] K. Lange and R. Carson, "EM reconstruction algorithms for emission and transmission tomography," J. Comp. Assist. Tomog., vol. 8, pp. 306-316, 1984

[5] K. Lange, "An overview of Bayesian methods in image reconstruction," Digital Image Synthesis and Inverse Optics - SPIE, vol. 1351, pp. $270-287,1990$.

[6] R. M. Lewitt and G. Muehllehner, "Accelerated iterative reconstruction for positron emission tomography based on the EM algorithm for maximum likelihood estimation," IEEE Trans. Med. Imaging, vol. 5, no. 1, 1986.

[7] J.-S. Liow, and S. C. Strother, "The convergence of object dependent resolution in maximum likelihood based tomographic image reconstruction," Physics in Medicine and Biology, Aug. 1992.

[8] J.-S. Liow and S. C. Strother, "Practical tradeoffs between noise quantitation, and number of iterations for maximum likelihoodbased reconstructions," IEEE Trans. Med. Imaging, vol. 10, no. 4, Dec. 1991.

[9] L. A. Shepp and Y. Vardi, "Maximum likelihood reconstruction for emission tomography," IEEE Trans. Med. Imaging, vol. 1, pp. $113-121,1982$.

[10] D. L. Snyder, M. I. Miller, L. J. Thomas, and D. G. Politte, "Noise and edge artifacts in maximum-likelihood reconstruction for emission tomography," IEEE Trans. Med. Imaging, vol. 6, pp. 228-238, 1987.

[11] E. Veklerov and J. Llacer, "Stopping rule for the MLE algorithm based on statistical hypothesis testing," IEEE Trans. Med. Imaging, vol. 6, pp. 313-319, 1987. 